\title{
TITAN \\ Exploring an Earthlike World
}

Second Edition 
Series Editor: F. W. Taylor (Oxford Univ., UK)

Vol. 1: TITAN: The Earth-Like Moon

Athena Coustenis \& Fredric W. Taylor

Vol. 2: Inverse Methods for Atmospheric Sounding: Theory and Practice Rodgers Clive D.

Vol. 3: Non-LTE Radiative Transfer in the Atmosphere M. López-Puertas \& F. W. Taylor

Vol. 4: TITAN: Exploring an Earthlike World (2nd Edition) Athena Coustenis \& Fredric W. Taylor 
Series on Atmospheric, Oceanic and Planetary Physics - Vol. 4

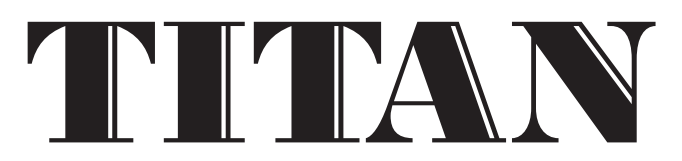

\title{
Exploring an Earthlike World
}

\author{
Second Edition
}

\section{Athena Coustenis}

Paris-Meudon Observatory

\section{Fredric W Taylor}

University of Oxford

With illustrations by D J Taylor 


\section{Published by}

World Scientific Publishing Co. Pte. Ltd.

5 Toh Tuck Link, Singapore 596224

USA office: 27 Warren Street, Suite 401-402, Hackensack, NJ 07601

UK office: 57 Shelton Street, Covent Garden, London WC2H 9HE

\section{British Library Cataloguing-in-Publication Data}

A catalogue record for this book is available from the British Library.

\section{Series on Atmospheric, Oceanic and Planetary Physics - Vol. 4 \\ TITAN (2nd Edition) \\ Exploring an Earthlike World}

Copyright (C) 2008 by World Scientific Publishing Co. Pte. Ltd.

All rights reserved. This book, or parts thereof, may not be reproduced in any form or by any means, electronic or mechanical, including photocopying, recording or any information storage and retrieval system now known or to be invented, without written permission from the Publisher.

For photocopying of material in this volume, please pay a copying fee through the Copyright Clearance Center, Inc., 222 Rosewood Drive, Danvers, MA 01923, USA. In this case permission to photocopy is not required from the publisher.

ISBN-13 978-981-270-501-3

ISBN-10 981-270-501-5

Typeset by Stallion Press

Email: enquiries@stallionpress.com

Printed in Singapore. 
Prologue ........................ xiii

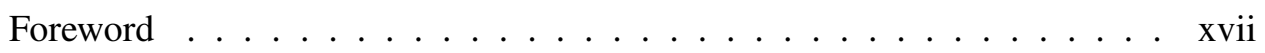

1. Introduction 1

1.1 Early History . . . . . . . . . . . . . . . . . . . . 1

1.2 Titan in Mythology . . . . . . . . . . . . . . . 5

1.3 Space Exploration of the Solar System . . . . . . . . . . . 8

1.4 The 20th Century, Before Voyager . . . . . . . . . . . . . . 13

2. The Voyager Missions to Titan 16

2.1 Space Missions to the Saturnian System . . . . . . . . . . . 16

2.2 Voyager Observations of Titan . . . . . . . . . . . 20

2.3 Atmospheric Bulk Composition . . . . . . . . . . . . 22

2.4 Vertical Temperature Structure . . . . . . . . . . . . . . 22

2.5 Energy Balance and the Temperature Profile in the Thermosphere . . . . . . . . . . . . . . 26

2.6 Atmospheric Composition . . . . . . . . . . . . . 28

2.7 Photochemistry . . . . . . . . . . . . . . . 31

2.8 Cloud and Haze Properties . . . . . . . . . . . . . . . . . . 34

2.9 Speculations on the Surface and Landscape of Titan

2.10 The Aftermath of Voyager . . . . . . . . . . . . 36

3. Observations of Titan from the Earth 38

3.1 Introduction . . . . . . . . . . . . . . . . 38

3.2 Space Observatories . . . . . . . . . . . . . . . . 38

3.2.1 Hubble Space Telescope . . . . . . . . . . . . . . . . 39

3.2.2 The James Webb Space Telescope . . . . . . . . . . . . 40

3.2.3 Infrared Space Observatory _. . . . . . . . . . . . . 41

3.3 Ground-Based Observatories . . . . . . . . . . . . . . . . . . 44

3.3.1 Mauna Kea Observatories . . . . . . . . . . . . . . . 44

3.3.2 The European Southern Observatories . . . . . . . . . 46 


\subsubsection{The University of Arizona and Steward Observatory} Telescopes . . . . . . . . . . . . . . 48

3.3.4 Radio Astronomy . . . . . . . . . . . . . . . . . . . . . 49

3.4 Earth-Based Studies of Titan . . . . . . . . . . . . . . . 52

3.4 .1 Occultations of Titan . . . . . . . . . . . . 52

3.4.2 The Radar Search for Oceans, Seas or Lakes . . . . . . 54

3.4.3 Spectroscopic Measurements of Titan's Albedo . . . . . 57

3.4.4 Imaging Titan's Atmosphere in the Near-Infrared . . . . 64

3.4.5 Imaging the Surface . . . . . . . . . . . . . . . 66

3.5 Ground-Based Observations and Cassini-Huygens . . . . . . 71

4. Cassini-Huygens: Orbiting Saturn and Landing on Titan 72

4.1 Introduction . . . . . . . . . . . . . . . . . 72

4.2 The Spacecraft and its Systems . . . . . . . . . . . . . . . 73

4.3 Scientific Objectives . . . . . . . . . . . . . . . . . 77

4.4 The Long History of the Cassini-Huygens Mission . . . . . . 79

4.5 Departure for the Saturnian System . . . . . . . . . . . . 82

4.6 Journey to Saturn and Orbit Insertion _ . . . . . . . . . . . 84

4.7 Huygens Descends onto Titan . . . . . . . . . . . . 85

4.8 Experiments and Payloads . . . . . . . . . . . . . . . . . 89

4.8.1 The Scientific Instruments on the Orbiter . . . . . . . . 89

4.8.2 The Scientific Instruments on the Probe . . . . . . . . . 99

4.9 Touring the Saturnian System _ . . . . . . . . . . . . . . 107

4.9.1 Observations of Saturn _. . . . . . . . . . . . 107

4.9.2 The Icy Satellites, and Saturn's Rings . . . . . . . . . . 109

4.9.3 Saturn's Magnetosphere and Titan . . . . . . . . . . . 112

4.10 Being Involved: Scientists and Instrument Providers . . . . . . . 113

4.11 Reaping the Benefits . . . . . . . . . . . . . . 115

$\begin{array}{ll}\text { Colour Plates } & 117\end{array}$

5. Titan's Atmosphere and Climate 129

5.1 The Climate on Titan . . . . . . . . . . . . . . . . . . . . . . . 129

5.1.1 Atmospheric Pressure Profile . . . . . . . . . . . . . 129

5.1.2 Atmospheric Thermal Structure . . . . . . . . . . . 131

5.1 .3 Troposphere . . . . . . . . . . . . . . . . 133

5.1 .4 Stratosphere . . . . . . . . . . . . . . 135

5.1 .3 Mesosphere . . . . . . . . . . . . . . . . . 136

5.1 .6 Thermosphere . . . . . . . . . . . . . . 136

5.1 .7 Exosphere . . . . . . . . . . . . . . . 136

5.2 Radiation in Titan's Atmosphere . . . . . . . . . . . . . . 137

5.2.1 Solar and Thermal Radiation . . . . . . . . . . . . . 137 
5.2.2 Energy Balance and Surface Temperature . . . . . . . 137

5.2.3 Model Temperature Profile . . . . . . . . . . . . . . . . 138

5.2.4 Radiative Equilibrium Temperature Profile . . . . . . . . 139

5.3 Remote Atmospheric Temperature Sounding . . . . . . . . . . . 141

5.4 Titan's Ionosphere and its Interaction with the Magnetosphere of Saturn . . . . . . . . . . . . . . . . . 144

5.5 Climate Change on Titan . . . . . . . . . . . . . . . . . 147

6. Chemistry and Composition $\quad 150$

6.1 Titan's Chemical Composition . . . . . . . . . . . . 150

6.2 The Bulk Composition of the Atmosphere . . . . . . . . . . . 154

6.3 Ionospheric Chemistry . . . . . . . . . . . . . . 155

6.4 Trace Constituents in the Neutral Atmosphere . . . . . . . . . . 157

6.4.1 Stratospheric Composition Measurements with Cassini . 160

6.4.2 Vertical Distributions . . . . . . . . . . . . . . . 175

6.4.3 Spatial Variations . . . . . . . . . . . . . . . . . . 177

6.4.4 Temporal Variations of the Trace Constituents . . . . . . 180

6.5 Photochemistry . . . . . . . . . . . . . . . 181

6.5.1 Hydrocarbons . . . . . . . . . . . . . . . . 185

$6.5 .2 \quad$ Nitriles . . . . . . . . . . . . . . . . . . . . 188

6.5.3 Oxygen Compounds . . . . . . . . . . . . . . . . . 189

6.5.4 Condensation Efficiencies . . . . . . . . . . . . . . 192

6.5.5 Aerosol Production . . . . . . . . . . . . . . . . 193

7. Clouds and Hazes 194

7.1 Introduction and Overview . . . . . . . . . . . . . . . . 194

7.2 Terrestrial Clouds and Precipitation _... . . . . . . . . 197

7.3 Visible Aspects of Titan's Haze . . . . . . . . . . . . . . . . . . 198

7.4 Size and Vertical Distribution of the Haze Particles _..... . . 201

7.4.1 Haze Vertical Profiles . . . . . . . . . . . . . . . . . 203

7.4.2 Haze Opacity Spatial Variations . . . . . . . . . . . . . 205

7.5 Tropospheric Condensate Clouds . . . . . . . . . . . . . . . . 206

7.6 Thermal and Dynamical Interactions with the Haze . . . . . . . 210

7.7 Observational Evidence on the Aerosol Composition . . . . . . 213

7.8 Laboratory Simulations of Haze Materials . . . . . . . . . . . 217

7.8.1 Chemical Composition of Tholins . . . . . . . . . . 218

7.8.2 Optical Properties of Tholins . . . . . . . . . . . . . . 221

7.9 Microphysical Models of Titan's Haze . . . . . . . . . . . . . 223

7.9.1 Organic Haze Production . . . . . . . . . . . . . . . . 223

7.9.2 Fractal Models and Scattering Properties of the Haze . . 227

7.10 Discussion and Conclusion . . . . . . . . . . . . . . . . . 229 
8. Atmospheric Dynamics and Meteorology 231

8.1 Introduction . . . . . . . . . . . . . . . . 231

8.2 Dynamics of Planetary Atmospheres . . . . . . . . . . 232

8.3 Titan's General Circulation . . . . . . . . . . . . . . 237

8.4 Zonal Motions . . . . . . . . . . . . . . 238

8.5 The Meridional Circulation . . . . . . . . . . . . . . 243

8.5.1 The Hemispherical Asymmetry . . . . . . . . . . . . . 244

8.5.2 The Polar Vortex . . . . . . . . . . . . . . . . . 245

8.6 Vertical Motions .................. . 247

8.7 Waves, Tides and Turbulence . . . . . . . . . . . . 250

8.8 The Weather Near the Surface . . . . . . . . . . . . . 251

8.9 Does Lightning Occur on Titan? . . . . . . . . . . . . . . 255

9. The Surface and Interior of Titan 258

9.1 Introduction .................... 258

9.2 Remote Sensing of the Surface . . . . . . . . . . . . . 259

9.3 Huygens Takes a Plunge ................ 261

9.4 Naming Distant New Places . . . . . . . . . . . . . 266

9.5 Evidence for Geological Activity . . . . . . . . . . . . . 269

9.5.1 Albedo Variations . . . . . . . . . . . . . . . . . 269

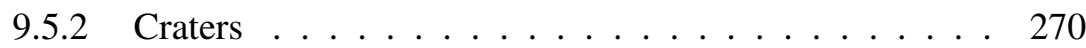

9.5.3 Mountains and Volcanoes ............ 273

9.5.4 Dunes ................... . . 274

9.5.5 Lakes .................... . . 275

9.6 The Nature and Composition of the Surface . . . . . . . . . . 277

9.7 The Interior of Titan . . . . . . . . . . . . . . 281

10. Titan's Origin and Evolution in the Solar System 286

10.1 Introduction . . . . . . . . . . . . . . . . . . . . 286

10.2 Relations Among Solar System Bodies . . . . . . . . . . . . . . 287

10.2.1 The Formation of the Solar System . . . . . . . . . . . 289

10.2.2 The Terrestrial Planets and Titan . . . . . . . . . . . . 290

10.2.3 Titan and the Outer Planets . . . . . . . . . . . . . . . 297

10.2.4 Titan and the Other Saturnian Satellites . . . . . . . . 304

10.2.5 Titan and Europa . . . . . . . . . . . . . . . . 318

10.2.6 Nitrogen Atmospheres in the Outer Solar System . . . . 318

10.3 Titan's Origin and Evolution . . . . . . . . . . . . . . . . . 321

10.3.1 Evolutionary Models for Titan's Atmosphere . . . . . . 324

10.3.2 Origin of the Atmospheric Components . . . . . . . . . 325

10.4 Titan and Life . . . . . . . . . . . . . . . . . . . . . . . . . . . . . . . 329

10.5 Open Questions . . . . . . . . . . . . . . . . 330 
11. Beyond Cassini/Huygens: The Future Exploration of Titan 332

11.1 Returning to Titan . . . . . . . . . . . . . . . 332

11.2 Titan as a Target of Astrobiological Interest . . . . . . . . . . 334

11.3 Science Drivers and Measurements Needed . . . . . . . . . . 335

11.4 Advanced Titan Mission Concepts . . . . . . . . . . . . . . . . 337

11.3 Technology Requirements . . . . . . . . . . . . . . . . . . . 339

11.6 Mission Architecture and Design . . . . . . . . . . . . . . . . . 339

11.7 Getting to Titan: Launch and Propulsion . . . . . . . . . . . 340

11.8 The Voyage of the Titania . . . . . . . . . . . . . . . . . 341

11.9 Explorers on Titan . . . . . . . . . . . . . . . 343

Glossary and Acronyms . . . . . . . . . . . . . . . . 350

References and Bibliography . . . . . . . . . . . . . . . 358

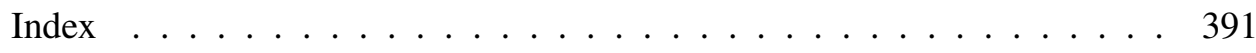


This page intentionally left blank 


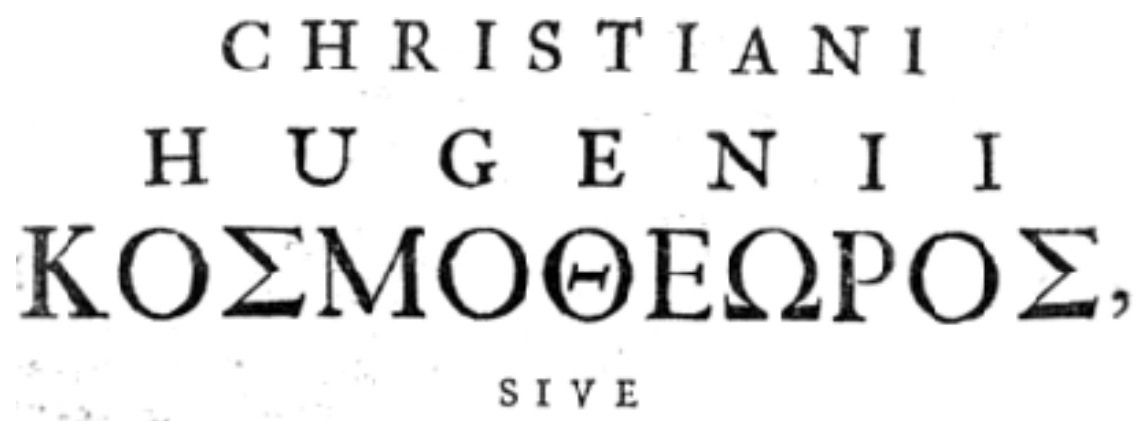

De Terris Coeleftibus, earumque ornatu,

C $a_{A} \mathbb{N}$ E C T U R .

की $A$ A

CONSTANTINUM HUGENU⿴囗十

Fratrem:

GULIELNO III. MAGNE BRITANNIEREGI, A SECRETIS.

A.159.

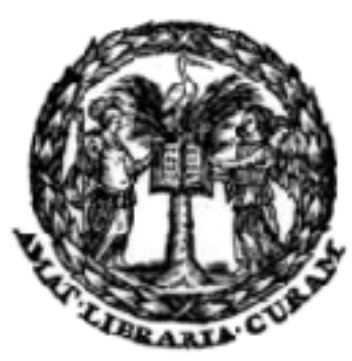

HAGE-COMITUM,

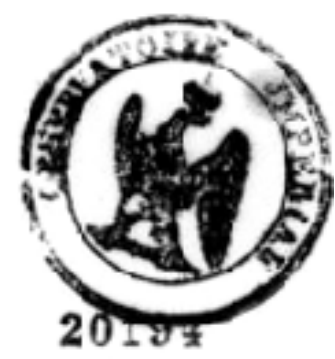

Apud Adrianum MOETJENS, Bibliopolam.

M. DC. X C YIII. 
This page intentionally left blank 
Humans have searched for another Earth practically since coming into existence, at least since we first began to realise that the points of light in the sky were large objects very far away, and the science of astronomy was born. In the modern era, every possible approach, from telescopes and space missions to philosophical arguments, is being used to probe the existence of Earthlike planets elsewhere in the universe. The ultimate question is whether there is another world sufficiently like ours, with the same temperature conditions and atmospheric composition, where we could live in comfort, or even where life could have developed in a form that we would readily recognise.

In the last thirty years or so, space missions and Earth-based telescopes have shown us that our own Solar System is mainly composed of uninhabited, and essentially uninhabitable, worlds. There is plenty of variety, ranging from giant balls of primordial gas with no solid surface (the outer planets) to objects that have no substantial atmosphere like Mercury and the minor planets; or like our Moon and almost all of the other satellites in the Solar System. Mars has enough atmospheric density to exhibit phenomena like polar caps, clouds, and dust storms, but the composition is mostly $\mathrm{CO}_{2}$, and therefore unfit for human respiration. Venus and Mercury are too hot and dry, and so on. Since the prospects for life are not good in our immediate neighbourhood, it is not surprising then that scientists have turned to the rest of the Universe, outside the Solar System.

Recently we have been witnessing the regular discoveries, through modern techniques ranging from adaptive optics and radio astronomy to gravitational microlensing, of protostellar disks, and of a rapidly increasing number of new extrasolar planets and planetary systems around distant stars like Beta Pictoris, Fomalhaut, 51 Pegasus, HD209458, and Tau Bootis. As we get better at searching, we increase our chances of finding another Earth some day, but it will not be nearby or easy to reach, even with robot probes. In our Solar System, in particular, the chances for life outside Earth, long ago considered excellent, receded as exploration revealed the details of the non-Earthlike environments on the most Earthlike planets, Mars and Venus. It made the surprise all the greater when we found, only relatively recently, that there is another place in the Solar System that does have an Earthlike atmosphere, albeit a very cold one. Strangely, this is not on any of the planets, but on a satellite, the only one among the dozen or so very large moons in the Sun's family for which this is true. 
Titan, Saturn's biggest moon, and (by a narrow margin) the second in size among the satellites in our Solar System, has been known for a long time to have a substantial atmosphere. The Catalan astronomer José Comas Solà claimed in 1908 to have observed limb darkening on Titan. This is the effect whereby the solar light reflected back to Earth by Titan's limb shows a stronger attenuation than that from its centre, which usually implies the presence of a substantial atmosphere. Confirmation came from spectroscopic observations by Gerard Kuiper in the 1940s, but it was not until the Voyager 1 spacecraft visited Titan in 1980 that the composition and the surface pressure were found to be so similar to those on Earth. Furthermore, complex organic chemistry is active there, producing multiple layers of orange-coloured haze, which render the atmosphere opaque in the optical range of wavelengths. It took a long time and a lot of effort to get a glimpse of the surface, and we still do not know today exactly what its composition is, except that the crust beneath must be mainly water ice.

To address the many questions asked about Titan over the centuries since its discovery, a series of space probes has been developed and dispatched towards this intriguing body. Pioneer 11 arrived first, in 1979, followed by Voyager 1 a year later. The scientific understanding of Titan as a planet-like object that emerged from the analysis of Voyager data was improved by ongoing ground-based observations, using increasingly more powerful optical and spectroscopic techniques, such as radar and adaptive optics, and advanced platforms on Earth-orbiting space observatories. At the same time as new data were acquired and studied, new theoretical models were being developed to account for these observations, new theories proposed and debated, and old or repeated measurements re-analysed. All of these have yielded formidable results, and posed new questions, over the past several years.

The latest envoy to Titan, a large and sophisticated international space mission called Cassini/Huygens, was launched in the year 1997. It arrived in July 2004, and started gathering new measurements from an orbit around Saturn that was designed to permit multiple Titan encounters. The Huygens probe descended in Titan's atmosphere on January 14, 2005 and recorded breathtaking data, revealing an astounding new world, and the most distant one to be landed on by a human-made machine.

The aim of this book is to bring together a general overview of our current understanding of all aspects of Titan, at a comprehensive and scientific level, but in terms basic enough to be mostly accessible to the non-specialist as well. We begin with a history of Titan studies, covering Earth-based facilities and programmes and leading into the early space missions. The Voyager mission, in particular, engulfed the scientific community with large amounts of data nearly twenty years ago, some of which is still being analyzed. The torrent of data from Cassini, with 40 times as many flybys, not to mention the Huygens in situ science, dwarfs even that from Voyager. We describe how, along with concurrent ground-based observations and theoretical modelling, Voyager, and now Cassini, have revolutionised our view of the outer Solar System. Titan's place in the Saturnian system, its structure and composition, its unique atmosphere, and the extent to which it really resembles the Earth, 
are the main themes. As a secondary objective, we hope to show the reader that the close look astronomers have been afforded since 2004 with their very expensive Cassini/Huygens space mission to the Saturnian system is paying dividends. An up-to-date synthesis of current Titan knowledge, and the remaining big questions, should give everyone the possibility to better appreciate the Cassini/Huygens discoveries.

We have tried to cover most of what is known about Titan today, and some informed speculation as well, while keeping the account as simple as possible. Our experience tells us that interest in Titan and its exploration, as evidenced by the huge popular reaction to the Cassini space mission, extends far beyond the small international group of professional planetary scientists. Thus, we have tried to make the book accessible to all, assuming only a basic familiarity with physics and astronomy. In the chapters that deal with more technical subjects, it has been necessary to use some more complicated concepts and words, in order not to leave out important knowledge or key questions. Where scientific terminology could not be avoided, the more specialised terms are defined in the text, in footnotes, and in the glossary, for the general reader. We hope that interested non-scientists will persevere as far as they can, and then move on to the next chapter where a more basic level is again resumed. For those who, on the other hand, want more detail, or wish to read about the original research of which this book is a summary, we include references and guides to further reading in a comprehensive bibliography at the end of the book.

Finally, we would like to acknowledge friends and colleagues who have helped with the text, either directly or by communicating their work on Titan. Particularly valuable inputs and comments on the draft manuscript came from David Luz, Patrick Irwin, Emmanuel Lellouch, Ralph Lorenz, Conor Nixon, Robert Samuelson, Tetsuya Tokano, Daniel Gautier and Thomas Widemann. For help with illustrations, proofreading and other helpful comments, we further thank Iannis Dandouras, Mathieu Hirtzig, Tom Krimigis, Panagiotis Lavvas, Alberto Negrão, Hasso Niemann and Véronique Vuitton. We are grateful to the EUROPLANET Consortium for funding part of our meetings to work on this project, and to the Observatoire de Paris for access to historical material. We are also grateful to many Cassini-Huygens scientists who provided guidance and information on their work. In particular we thank Dennis Matson and Jean-Pierre Lebreton, Cassini-Huyens project managers who kindly read the book in advance and wrote a foreword.

The original figures in this volume were drawn and montages created by Dr. D. J. Taylor, to whom we extend our deepest gratitude.

Athena Coustenis Fred W Taylor Paris and Oxford, April 2008 
This page intentionally left blank 


\section{Foreword}

Titan is Saturn's largest moon. It was discovered by the Dutch Astronomer Christian Huygens in 1655. For almost three centuries thereafter, Titan remained nothing more than a dot of light in the sky. Then, in 1994, Gerard Kuiper discovered that its atmosphere contained methane! It immediately became a world to explore and a destination in the space era.

Titan is shrouded by a thick atmosphere with a blanket of organic haze that hides the surface. The haze is a product of UV photolysis of methane in the upper atmosphere. In the early 80s, Voyager investigated Titan but was unable to see through to the surface. However, Voyager confirmed that the atmosphere served as a big chemical factory producing many complex organic compounds. This made Titan one of the most fascinating bodies in the solar system.

In 1999, while the international Cassini-Huygens mission was on its voyage to Saturn, the authors of this book published their first comprehensive review of the knowledge of Titan. They are two of the scientists most knowledgeable about this fascinating moon of Saturn. As a result of Cassini-Huygens' arrival around Saturn in 2004, and the Huygens probe landing on Titan's surface on January 14, 2005, a giant advance has been made in our understanding of Titan. Thus, it is timely to issue a new textbook. Athena Coustenis and Fred Taylor have come up with a superb revised version of their book. The new edition gives an excellent and up to date account of our knowledge about Titan. It provides a comprehensive review of this peculiar solar system object which bears many similarities to Earth albeit under very different conditions, where methane plays the role that water plays on Earth. The latest results are described and include Earth-based observations, laboratory work and modelling in addition to the Cassini-Huygens observations.

The book tells the history of the exploration of Titan. It includes the most recent ideas about the processes that govern Titan. It shows extremely well the synergy of in situ robotic observations and space-based and Earth-based telescopic observations. It also shows the importance of laboratory work and modelling. All of these approaches are needed to make progress in our understanding of Solar System objects. The book is an excellent reference for students with some general background in the field of planetary sciences and for new planetary scientists looking for a comprehensive book on Titan. After reading the book, you may decide to stick with Titan for the rest of your career! The authors of the book are currently active 
in the Cassini-Huygens mission and have been involved in Titan research for several decades. They have mentored young scientists who are now Titan researchers themselves.

Titan has become a well-known object in the Solar System. It is the most Earthlike world we know of. This book puts this extraordinary solar system object on our Solar System map for additional exploration. The closing chapter describes new concepts for the future exploration of Titan and gives us an irresistible invitation to return.

Jean-Pierre Lebreton ESA Huygens Project Scientist

Dennis Matson NASA Cassini Project Scientist 